\title{
Nasopharyngeal Carcinoma by AJCC v6 Stage
}

National Cancer Institute

\section{Source}

National Cancer Institute. Nasopharyngeal Carcinoma by A/CC v6 Stage. NCI Thesaurus.

Code C90522.

A term that refers to the staging of nasopharyngeal carcinoma according to the American Joint Committee on Cancer, 6th edition. 